\title{
Commentary: Thank you, sir, may I TAVI another: Procedural problems and postoperative complications in SURTAVI
}

\author{
Judson B. Williams, MD, MHS, ${ }^{a}$ and Jeffrey G. Gaca, MD
}

\footnotetext{
From the ${ }^{\mathrm{a}}$ Department of Cardiovascular and Thoracic Surgery, WakeMed Health and Hospitals, Duke University School of Medicine, Raleigh, NC; and ${ }^{\mathrm{b}}$ Department of Cardiovascular and Thoracic Surgery, Duke Health, Duke University School of Medicine, Durham, NC.

Disclosures: Authors have nothing to disclose with regard to commercial support.

Received for publication Nov 24, 2018; accepted for publication Nov 26, 2018; available ahead of print Jan 16 , 2019.

Address for reprints: Judson B. Williams, MD, MHS, Cardiovascular and Thoracic Surgery, WakeMed Health and Hospitals, Duke University School of Medicine, 3000 New Bern Ave, Suite 1100, Raleigh, NC 27610 (E-mail: judson.williams@duke.edu).

J Thorac Cardiovasc Surg 2019;158:729-30

$0022-5223 / \$ 36.00$

Copyright (C) 2018 by The American Association for Thoracic Surgery

https://doi.org/10.1016/j.jtcvs.2018.11.079
}

We congratulate the Surgical Replacement and Transcatheter Aortic Valve Implantation (SURTAVI) Trial Causes of Death Working Group on a fantastic presentation of the first year of data after randomization, presented by Amrane and colleagues ${ }^{1}$ in this issue of the Journal. Readers will recall that the industry-funded (Medtronic, Minneapolis, Minn) SURTAVI trial enrolled 1660 intermediate-risk patients from June 2012 to June 2016 to receive TAVR with self-expanding valves or surgical aortic valve replacement (SAVR). ${ }^{2}$ For the article of Amrane and colleagues, ${ }^{1}$ in addition to trial-based clinical event classification of deaths, Drs Amrane and Reardon independently reviewed each death themselves. This welcome step enabled a hierarchic approach to cause of death and achieved a high-quality data granularity not often seen with secondary analyses of clinical trial data.

As TAVR is becoming a ubiquitous procedure, a key message from this analysis is that procedural problems are the most common cause of death after TAVR, demonstrating that we still have work to do to improve on our use of this relatively new technology. A multivariate analysis of predictors of death was not possible because of the low event rate in each period. Even at the select European and North American sites chosen for SURTAVI enrollment, however, procedural problems-ventricular perforation, valve malposition, and paravalvular leak-are clearly identified as the most common cause of death for patients undergoing TAVR.

In this setting, standardized training for TAVR sites appears increasingly essential. Further improvements in equipment and refinements of implantation technique may also lower the risk. Amrane and colleagues ${ }^{1}$ discuss several advances with the self-expanding platform, including newer generation devices (representing 16\% of those used in SURTAVI) that allow smaller access vessel size $(\geq 5-5.5 \mathrm{~mm})$ and are recapturable, to facilitate

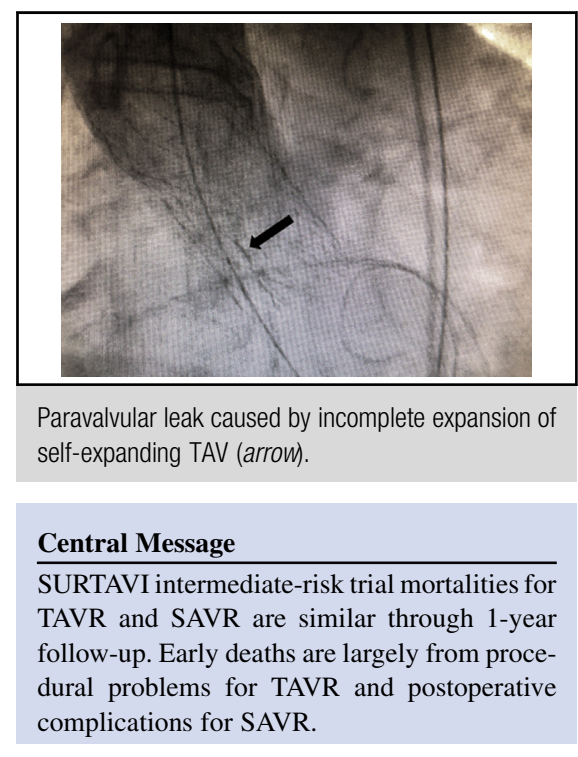

See Article page 718.

repositioning. In contradistinction, the predominant cause of death for patients undergoing SAVR is postoperative complications, highlighting the more invasive and more mature nature of that technology. Not discussed, but also of vital importance, are the advances being made to mitigate postoperative complications after SAVR, including enhanced recovery after cardiac surgery protocols.

TAVR was initially tested in extreme- and high-risk patient groups, for whom its superiority to SAVR has been well documented. ${ }^{4}$ As TAVR is applied to lower-risk populations, such as the intermediate-risk population in this analysis, such factors as device durability, patientprosthesis mismatch, pacemaker implantation, and paravalvular leak will greatly increase in importance. Follow-up much longer than 1 year will therefore be vital to answer the question of who is best treated with TAVR. We look forward to more detailed analyses from the SURTAVI Trial Causes of Death Working Group of patient-prosthesis mismatch, because even severe patient-prosthesis mismatch did not influence survival through 1 year for either the TAVR or SAVR group. We also look forward to more detailed analysis from this group of experts with regard to the effect of pacemaker implantation on long-term results.

The data presented by Amrane and colleagues ${ }^{1}$ are nonetheless highly informative. Again, we congratulate the 
SURTAVI Trial Causes of Death Working Group on a superb presentation and look forward to further results from the important SURTAVI randomized trial.

\section{References}

1. Amrane H, Deeb GM, Popma JJ, Yakubov SJ, Gleason TG, Van Mieghem NM, et al; the SURTAVI Trial Causes of Death Working Group. Causes of death in intermediate risk patients: the randomized surgical replacement and transcatheter aortic valve implantation trial. J Thorac Cardiovasc Surg. 2019;158:718-28.e3.
2. US National Library of Medicine ClinicalTrials.gov. Safety and Efficacy Study of the Medtronic CoreValve ${ }^{\circledR}$ System in the Treatment of Severe, Symptomatic Aortic Stenosis in Intermediate Risk Subjects Who Need Aortic Valve Replacement (SURTAVI). Available at: https://clinicaltrials.gov/ct2/show/ NCT01586910. Accessed December 21, 2018.

3. ERAS Cardiac Surgery. Available at: www.erascardiac.org. Accessed December 21, 2018.

4. Tice JA, Sellke FW, Schaff HV. Transcatheter aortic valve replacement in patients with severe aortic stenosis who are at high risk for surgical complications: summary assessment of the California technology assessment forum. $J$ Thorac Cardiovasc Surg. 2014;148:482-91.e6. 